\title{
Vacuum Sealing Drainage Treatment of Multi-Drug-Resistant Enterobacter Cloacae Wound Infection in a Diabetic Patient: A Case Report and Review of the Literature
}

\author{
Xiao-hua Pan ${ }^{1 *}$, Xiao-min Wu ${ }^{1,2}$, Xin-shen Jin ${ }^{1}$, Shu-yun Xiang ${ }^{1}$ and Cong-cong S ${ }^{1}$ \\ ${ }^{1}$ Department of Orthopaedics and Traumatology, BaoAn Hospital affiliated to Southern Medical University \& ShenZhen 8th People Hospital \\ ${ }^{2}$ Shenzhen Research Institute of Trauma Rescue and Regenerative Medicine
}

*Corresponding Author: Pan Xiao-hua, Department of Orthopaedics and Traumatology, BaoAn People Hospital affiliated to Southern Medical University \& ShenZhen 8th People Hospital, ShenZhen, PR China, Tel: 0755-27788311-3953; Email: 568282414@qq.com

Received date: March 06, 2017; Accepted date: March 27, 2017; Published date: March 31, 2017

Copyright: ( 2017 Pan X, et al. This is an open-access article distributed under the terms of the Creative Commons Attribution License, which permits unrestricted use, distribution, and reproduction in any medium, provided the original author and source are credited.

\begin{abstract}
Diabetic foot ulcers differ from non-diabetic trauma, as diabetic patients have dysfunctional wound healing owing to changes in the microcirculation. Herein, we report the case of a 48-year-old man with a 4-year history of uncontrolled type 2 diabetes. The patient had developed a 3-cm-wide ulcer deep to the phalanx on the big toe of the right foot 3 weeks ago, and the ulcer became infected with multi-drug-resistant Enterobacter cloacae 1 week later. We performed thorough wound debridement combined with Vacuum Sealing Drainage (VSD) and intravenous immunoglobulin administration without antibiotic therapy. This treatment strategy reduced the size of the ulcer. Three weeks later, antibiotic-susceptible Staphylococcus aureus was cultured from the wound tissue instead of Enterobacter cloacae, and so, the patient was administered antibiotics. The patient recovered well after this simple procedure followed by antibiotic therapy.
\end{abstract}

Keywords: Diabetic food infection; VSD; Multi-drug resistance; Enterobacter cloacae

\section{Introduction}

Diabetes affects almost 171 million people worldwide, and this number is expected to increase to 366 million by 2030 [1]. Approximately $15 \%$ of diabetic patients will develop a foot ulcer during their lifetime [2]. Diabetic foot ulcers are induced by not only necrosis of the deep tissues due to neurological or vascular disease of the lower limbs but also wound infection [3]. Long-term hyperglycemia alters the microcirculation, which impedes wound healing [4]. Such ulcers are vulnerable to infections. The wound exudate in such ulcers contains protein-rich lymph and serves as a medium for microbial proliferation [5]. Furthermore, when infection does occur, it spreads rapidly. All these changes are attributable to low immunity, which is related with diabetes [6].

Empirical antimicrobial therapy is the main cause of antibiotic resistance. Although Enterobacter cloacae is not a prevalent pathogen in diabetic foot ulcers, multi-drug-resistant Enterobacter spp. have increasingly emerged as a cause of hospital-acquired infections [7]. These gram-negative bacteria have been reported to be important causes of opportunistic and multi-drug-resistant infections in humans during the last three decades [8]. About $10 \%$ of patients with Enterobacter infections are non-responsive to treatment [9]. Here, we present one such case of that was successfully treated using an alternative treatment along with antibiotic therapy.

\section{Case Presentation}

A 48-year-old man with type 2 diabetes was transferred to our department with chief complaints of a non-healing ulcer resulting from a toe injury sustained during an accidental puncture 3 weeks prior. The patient had had diabetes for approximately 4 years, and his blood sugar level was not well controlled. He underwent wound debridement and was treated with intravenous antibiotics for 2 weeks, without any improvement. In fact, the foot ulcer deteriorated further, and amputation was recommended by the medical department.

Upon examination in our department, we found that the ulcer was about $3 \mathrm{~cm}$ wide, deep to the phalanx on the big toe of the right foot, and filled with pus. The lesion was pale, and the skin around the wound was puffy (Figure 1A). Further clinical examination and medical imaging revealed that anteroposterior and lateral radiographs of the right foot and the arterial and venous circulation in the lower extremity were normal. Laboratory examinations showed the following: white blood cell count, 10,230/ $\mu \mathrm{L}, 59.1 \%$ neutrophils, and erythrocyte-sedimentation rate, 17. After 1 week of intravenous antibiotic treatment, bacterial cultures and drug-sensitivity tests of the wound specimens revealed multi-drug-resistant Enterobacter cloacae (Table 1).

\begin{tabular}{|l|l|l|}
\hline Antibiotic & Susceptibility & $\begin{array}{l}\text { MIC } \\
(\mathrm{mg} / \mathrm{L})\end{array}$ \\
\hline Amikacin & $\mathrm{R}$ & $>32$ \\
\hline Amoxicillin/Clavulanate & $\mathrm{R}$ & $>16 / 8$ \\
\hline Ampicillin & $\mathrm{R}$ & $>16$ \\
\hline Aztreonam & $\mathrm{R}$ & $>16$ \\
\hline Cefazolin & $\mathrm{R}$ & $>16$ \\
\hline Cefepime & $\mathrm{R}$ & $>16$ \\
\hline Cefotaxime & $\mathrm{R}$ & $>32$ \\
\hline
\end{tabular}


Citation: Pan X, Wu X, Jin X, Xiang S, Su C (2017) Vacuum Sealing Drainage Treatment of Multi-Drug-Resistant Enterobacter Cloacae Wound Infection in a Diabetic Patient: A Case Report and Review of the Literature. J Nutr Disorders Ther 7: 210. doi: 10.4172/2161-0509.1000210

Page 2 of 3

\begin{tabular}{|l|l|l|}
\hline Cefoxitin & $\mathrm{R}$ & $>16$ \\
\hline Ceftazidime & $\mathrm{R}$ & $>16$ \\
\hline Ceftriaxone & $\mathrm{R}$ & $>32$ \\
\hline Cefuroxime & $\mathrm{R}$ & $>16$ \\
\hline Ciprofloxacin & $\mathrm{R}$ & $>2$ \\
\hline Gentamicin & $\mathrm{R}$ & $>8$ \\
\hline Imipenem & $\mathrm{R}$ & $>8$ \\
\hline Levofloxacin & $\mathrm{R}$ & $>4$ \\
\hline Meropenem & $\mathrm{R}$ & $>8$ \\
\hline Piperacillin/Tazobactam & $\mathrm{R}$ & $>64$ \\
\hline Piperacillin & $\mathrm{R}$ & $>64$ \\
\hline Tetracycline & $\mathrm{R}$ & $>8$ \\
\hline Ticarcillin/Clavulanic acid & $\mathrm{R}$ & $>64$ \\
\hline Tobramycin & $\mathrm{R}$ & $>8$ \\
\hline Trimethoprim/ & $\mathrm{R}$ & $>2 / 38$ \\
\hline Sulfamethoxazole & & \\
\hline R: resistant; MIC, median inhibitory concentration & & \\
\hline
\end{tabular}

Table 1: Antibiotic sensitivity of Enterobacter cloacae cultured from wound tissue specimens

With the patient's consent, a decision was made to perform wound debridement and thorough cleaning and irrigation of the wound with hydrogen peroxide without any antibiotic. A sterile and disposable scalpel was used to remove all necrotic tissue. . The lesion was packed with sterile gauze soaked with povidone-iodine. Subsequently, the wound surface was enclosed with vacuum sealing drainage (VSD), and intravenous human immunoglobulin therapy was administered for 2 weeks. After 20 days of treatment, bacterial culture of the wound tissue showed Enterobacter cloacae sensitive to amikacin and gentamicin. One week later, Staphylococcus aureus isolates that were sensitive to most of the antibiotics tested were detected instead of Enterobacter cloacae. Considering the culture results, we treated the patient with gentamicin, and the wound healed well 10 days later without any infection (Figure 1B). The patient was discharged in good condition.

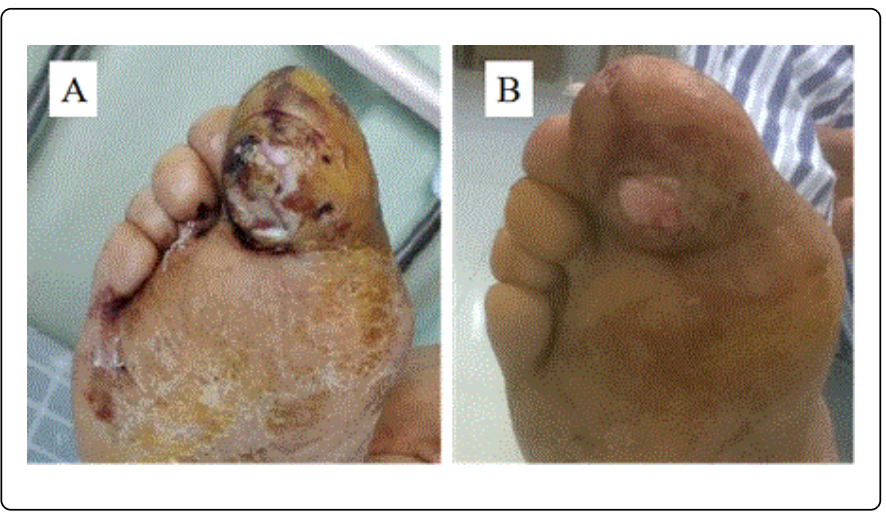

Figure 1: The affected skin and necrotic tissue (A) were debrided, and the wound healed well (B).

\section{Discussion}

Enterobacter cloacae, a well-known member of the family Enterobacteriaceae, are a common rod-shaped, non-spore-forming, facultative anaerobic gram-negative organism. It is not only widely encountered in the environment but also is a common nosocomial pathogen causing bacteremia, endocarditis, septic arthritis, and osteomyelitis. This is because the bacterium possesses redundant regulatory cascades that protect it by controlling membrane permeability and the expression of detoxifying enzymes involved in antibiotic degradation/inactivation. The production of constitutive AmpC $\beta$-lactamase is the main reason for its intrinsic resistance to ampicillin, amoxicillin-clavulanic acid, and cephalothin [10]. New Delhi metallo-beta-lactamase-1 (NDM-1) is the latest resistance enzyme identified in Enterobacter cloacae in 2008 [11]. Band et al. reported that the histidine kinase PhoQ is necessary for the development of a colistin-resistant subpopulation of Enterobacter cloacae [9]. The data also highlight that lysozyme, cathelin-related antimicrobial peptide (CRAMP), and $\mathrm{H} 2 \mathrm{O} 2$ are specific host innate immune components that increase the antibiotic-resistant subpopulation during infection [9]. A considerable body of literature has shown that enzymatic barrier, membrane barrier, and mutation render this species the main contributor to the multi-drug-resistant infection problem.

It is well-known that all infected wounds must be treated with antibiotics; however, the wound care provided is often insufficient. VSD is an ideal technique to induce healing in various types of tissue injuries, such as deep wounds, large skin defects, and even severe infections $[12,13]$. This negative-pressure device facilitates the suction of seepage, pus, and necrotic tissue from wounds to accelerate wound healing and ameliorate infection [14]. Nevertheless, its limited ability to kill microorganisms means that the patient's immunity against organisms must be concomitantly enhanced. White blood cell count, neutrophil-to-lymphocyte ratio, and fasting blood sugar level are significantly correlated with the activity of the immune system in type 2 diabetes [15]. Our patient had uncontrolled blood sugar levels for 4 years, and developed a multi-drug-resistant infection after sustaining an injury. Human immunoglobulin therapy is widely used in diabetic patients, as it increases antigen phagocytosis, natural killer cell activity, cell clones, and antibody response [16]. Several studies have revealed a relationship between systemic inflammation and insulin resistance [17].

The overuse and inappropriate use of antibiotics are the most important causes of antimicrobial resistance [18]. In this case, we performed wound debridement first to prevent deterioration, and then applied VSD and administered intravenous immunoglobulins to promote wound healing. This treatment strategy effectively resolved the multi-drug-resistant infection of the diabetic foot ulcer in our patient.

\section{References}

1. Wild S, Roglic G, Green A, Sicree R, King H (2004) Global prevalence of diabetes: estimates for the year 2000 and projections for 2030. Diabetes Care 27: 1047-1053. 
Citation: Pan X, Wu X, Jin X, Xiang S, Su C (2017) Vacuum Sealing Drainage Treatment of Multi-Drug-Resistant Enterobacter Cloacae Wound Infection in a Diabetic Patient: A Case Report and Review of the Literature. J Nutr Disorders Ther 7: 210. doi: $10.4172 / 2161-0509.1000210$

Page 3 of 3

2. Motley TA, Gilligan AM, Lange DL, Waycaster CR, Dickerson JJ (2015) Cost-effectiveness of clostridial collagenase ointment on wound closure in patients with diabetic foot ulcers: economic analysis of results from a multicenter, randomized, open-label trial. J Foot Ankle Res 8: 7.

3. Game F (2016) Classification of diabetic foot ulcers. Diabetes Metab Res Rev 32 Suppl 1: 186-194.

4. Kandemir O, Akbay E, Sahin E, Milcan A, Gen R (2007) Risk factors for infection of the diabetic foot with multi-antibiotic resistant microorganisms. J Infect 54: 439-445.

5. Lin CT, Ou KW, Chang SC (2013) Diabetic foot ulcers combination with lower limb lymphedema treated by staged charles procedure: case report and literature review. Pak J Med Sci 29: 1062-1064.

6. Fejfarova V, Jirkovska A, Dubsky M, et al. (2016) An Alteration of Lymphocytes Subpopulations and Immunoglobulins Levels in Patients with Diabetic Foot Ulcers Infected Particularly by Resistant Pathogens. J Diabetes Res 2016: 2356870.

7. Turhan V, Mutluoglu M, Acar A, et al. (2013) Increasing incidence of Gram-negative organisms in bacterial agents isolated from diabetic foot ulcers. J Infect Dev Ctries 7: 707-712.

8. Davin-Regli A, Pages JM (2015) Enterobacter aerogenes and Enterobacter cloacae; versatile bacterial pathogens confronting antibiotic treatment. Front Microbiol 6: 392.

9. Band VI, Crispell EK, Napier BA, et al. (2016) Antibiotic failure mediated by a resistant subpopulation in Enterobacter cloacae. Nat Microbiol 1: 16053.

10. Zaher A, Cimolai N (1997) ERIC-PCR typing profiles of Enterobacter cloacae are stable after development of advanced cephalosporin resistance. Int J Antimicrob Agents 9: 165-167.
11. Khan AU, Nordmann P (2012) NDM-1-producing Enterobacter cloacae and Klebsiella pneumoniae from diabetic foot ulcers in India. J Med Microbiol 61: 454-456.

12. Liu X, Liang J, Zao J, et al. (2016) Vacuum Sealing Drainage Treatment Combined with Antibiotic-Impregnated Bone Cement for Treatment of Soft Tissue Defects and Infection. Med Sci Monit 22: 1959-1965.

13. Ni J, Liu H, Liu X, et al. (2015) Vacuum Sealing Drainage as Treatment of Severe Buttocks and Perianal Infection: A Case Report and Review of the Literature (Care-Compliant). Medicine (Baltimore) 94: e1766.

14. Li RG, Yu B, Wang G, et al. (2012) Sequential therapy of vacuum sealing drainage and free-flap transplantation for children with extensive softtissue defects below the knee in the extremities. Injury 43: 822-828.

15. Eftekharian MM, Karimi J, Safe M, Sadeghian A, Borzooei S, Siahpoushi E (2016) Investigation of the correlation between some immune system and biochemical indicators in patients with type 2 diabetes. Hum Antibodies 24: 25-31.

16. Panto F, Giordano C, Amato MP, et al. (1990) The influence of high dose intravenous immunoglobulins on immunological and metabolic pattern in newly diagnosed type I diabetic patients. J Autoimmun 3: 587-592.

17. Shoelson SE, Lee J, Goldfine AB (2006) Inflammation and insulin resistance. J Clin Invest 116: 1793-1801.

18. Joshi N, Caputo GM, Weitekamp MR, Karchmer AW (1999) Infections in patients with diabetes mellitus. N Engl J Med 341: 1906-1912. 